\title{
Yania halotolerans gen. nov., sp. nov., a novel member of the suborder Micrococcineae from saline soil in China
}

Correspondence

Cheng-Lin Jiang

lihxu@ynu.edu.cn or

liact@yahoo.com

\author{
Wen-Jun Li, ${ }^{1}$ Hua-Hong Chen, ${ }^{1}$ Ping Xu, ${ }^{1}$ Yu-Oin Zhang, ${ }^{1}$ \\ Peter Schumann, ${ }^{2}$ Shu-Kun Tang, ${ }^{1}$ Li-Hua $X u^{1}$ and Cheng-Lin Jiang ${ }^{1}$
}

\author{
${ }^{1}$ Key Laboratory for Microbial Resources of the Ministry of Education, Yunnan Institute of \\ Microbiology, Yunnan University, Kunming, Yunnan, 650091, P.R. China \\ ${ }^{2}$ DSMZ - Deutsche Sammlung von Mikroorganismen und Zellkulturen GmbH, Mascheroder \\ Weg 1b, D-38124 Braunschweig, Germany
}

Phylogenetic analysis based on 16S rRNA gene sequence data is currently one of the most effective methods for delineation of bacteria. For descriptions of actinomycete genera or species, organisms should also be characterized with respect to a rich spectrum of chemotaxonomic properties, such as profiles of fatty acids, polar lipids, peptidoglycan amino acids, isoprenoid quinones and, less frequently, polyamines (Stackebrandt \& Schumann, 2000). For classification at higher taxonomic ranks of the classis Actinobacteria, i.e. families or suborders, $16 \mathrm{~S}$ rDNA signature nucleotide data should also be used (Stackebrandt et al., 1997; Stackebrandt \& Schumann, 2000). Both a separate phylogenetic position and the emergence of novel signature patterns are indicative of a novel higher taxon (Stackebrandt \& Schumann, 2000).

Until now, 13 families with validly published names have been described in the suborder Micrococcineae, including the family Micrococcaceae Pribram 1929 emend. Stackebrandt et al. 1997, the family Brevibacteriaceae Breed 1953 emend.

The GenBank/EMBL/DDBJ accession number for the $16 \mathrm{~S}$ rDNA sequence of strain YIM $70085^{\top}$ is AY228479.
Stackebrandt et al. 1997 and other families of Gram-positive cocci and rods. In this study, we report the results of polyphasic identification of a halotolerant actinobacterial strain, designated strain YIM $70085^{\mathrm{T}}$, that was isolated from a saline soil sample in Xinjiang Province, west China. The strain grew optimally in media that contained $10 \% \mathrm{KCl}$, $\mathrm{NaCl}$ or $\mathrm{MgCl}_{2} \cdot 6 \mathrm{H}_{2} \mathrm{O}$. Based on the results of chemotaxonomic analyses, this strain is distinct from members of all actinomycete families in the suborder Micrococcineae. $16 \mathrm{~S}$ rDNA sequence comparison revealed that strain YIM $70085^{\mathrm{T}}$ represents a distinct lineage within the suborder Micrococcineae, exhibiting $<94 \cdot 3 \%$ similarity to its phylogenetic neighbours. In addition, its $16 \mathrm{~S}$ rDNA signature nucleotides are different from those of members of other families in the suborder Micrococcineae. Our results indicate that the isolate should be placed as a novel member of the suborder Micrococcineae: Yania halotolerans gen. nov., sp. nov. However, as the novel genus and species is represented only by a single strain, affiliation to a novel higher taxon should await phylogenetic analyses of further isolates of this genus. Strain YIM $70085^{\mathrm{T}}$ has been deposited in the China Center for Type Culture Collection as strain CCTCC AA001023 ${ }^{\mathrm{T}}$ and in the Deutsche Sammlung von Mikroorganismen und Zellkulturen as DSM $15476^{\mathrm{T}}$. 


\section{Micro-organisms and culture conditions}

Strain YIM $70085^{\mathrm{T}}$ was isolated by using the dilution plating method from a saline soil sample from Xinjiang Province, west China. The medium used for selective isolation was glycerol/asparagine agar (ISP 5 medium; Shirling \& Gottlieb, 1966) that was supplemented with $15 \% \mathrm{KCl}(\mathrm{w} / \mathrm{v})$ and incubated at $28^{\circ} \mathrm{C}$ for about 2 weeks. The strain was maintained on potato agar or ISP 5 agar slants that contained $10 \% \mathrm{KCl}(\mathrm{w} / \mathrm{v})$ at $4{ }^{\circ} \mathrm{C}$ and as glycerol suspensions $(20 \%$, $\mathrm{v} / \mathrm{v}$ ) at $-20^{\circ} \mathrm{C}$. Biomass for chemical and molecular systematic studies was obtained by cultivation in flasks of modified ISP 5 medium $(10 \% \mathrm{KCl}, \mathrm{w} / \mathrm{v}$; $\mathrm{pH} 7 \cdot 0)$ that were shaken (at about 150 r.p.m.) at $28^{\circ} \mathrm{C}$ for 1 week.

\section{Morphological observations}

Morphological properties were examined by light microscopy with a model $\mathrm{BH}-2$ microscope (Olympus) and by transmission electron microscopy with a model $\mathrm{H}-800$ transmission electron microscope (Hitachi). Colour determination was done with colour chips from the ISCC-NBS Color Charts Standard Samples no. 2106 (Kelly, 1964). Morphological features were observed on glycerol/asparagine agar medium that was supplemented with $10 \%(\mathrm{w} / \mathrm{v})$ $\mathrm{KCl}$.

Morphological observation of a $24-48$ h culture of strain YIM $70085^{\mathrm{T}}$, grown on glycerol/asparagine agar (with $10 \%$ $\mathrm{KCl}$ ), revealed that cells were non-spore-forming, were coccoid or oval, were about $0 \cdot 4-0 \cdot 7 \mu \mathrm{m}$ in diameter and occurred singly or in clusters (Fig. 1). Colonies reached maximum size ( $2 \mathrm{~mm}$ in diameter) after 1 week incubation at $28^{\circ} \mathrm{C}$. They were yellow, circular, lubricious and opaque.

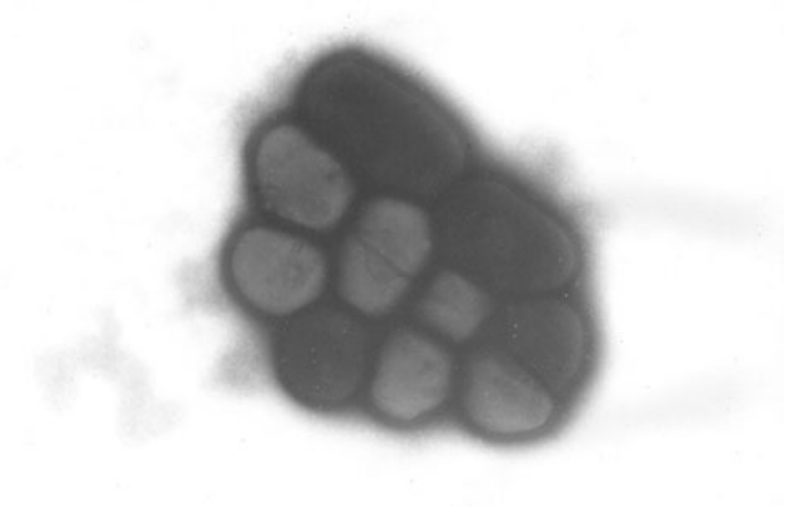

Fig. 1. Transmission electron micrograph of strain YIM $70085^{\top}$ grown on glycerol/asparagine agar (ISP 5 medium) with $10 \%$ $\mathrm{KCl}(\mathrm{w} / \mathrm{v})$ for 2 days at $28^{\circ} \mathrm{C}$. Bar, $0.5 \mu \mathrm{m}$.

\section{Metabolic properties}

Gram-staining was carried out on $24-48$ h cultures. Catalase activity was determined by production of bubbles after the addition of a drop of $3 \% \mathrm{H}_{2} \mathrm{O}_{2}$. All physiological and biochemical tests were performed at $28^{\circ} \mathrm{C}$. Carbon source utilization tests, sugar fermentation tests and qualitative enzyme tests were carried out in microtitre plates, according to Kämpfer et al. (1991). Some physiological properties were tested by using the API Coryne system and an API ID32 E test kit (bioMérieux).

Strain YIM $70085^{\mathrm{T}}$ was Gram-positive and aerobic; it utilized almost all carbon sources tested, but acid was produced only from glucose, maltose, sucrose and fructose. Its physiological and biochemical properties are given in detail in the species description.

\section{Chemotaxonomic properties}

Sugar analysis of whole-cell hydrolysates followed procedures that were described by Stanek \& Roberts (1974). Purified peptidoglycan preparations were obtained by the method of Schleifer \& Kandler (1972). Amino acids and peptides in cell-wall hydrolysates were analysed by twodimensional ascending TLC on cellulose plates, using the solvent systems of Schleifer \& Kandler (1972). The $\mathrm{N}$-terminal amino acid of the interpeptide bridge was determined by dinitrophenylation, as described by Schleifer (1985). Molar ratios of amino acids were determined by GC and GC-mass spectrometry of $N$-heptafluorobutyryl amino acid isobutyl esters (MacKenzie, 1987). Analysis of enantiomers of peptidoglycan amino acids was performed by GC of $\mathrm{N}$-pentafluoropropionyl amino acid isopropyl esters (Frank et al., 1980) on an L-Chirasil-Val column (Macherey-Nagel) as described by Groth et al. (1997).

Polar lipids were extracted, examined by two-dimensional TLC and identified by using published procedures (Minnikin et al., 1979; Collins \& Jones, 1980). Menaquinones were isolated by using the method of Collins et al. (1977) and analysed by HPLC (Groth et al., 1997). Analysis of the whole-cell fatty acid pattern followed the MIDI system (Microbial ID) (Kroppenstedt, 1985; Meier et al., 1993).

Enantiomeric analysis of peptidoglycan amino acids revealed equal amounts of L- and D-Glu. As D-Glu occupies position 2 of the peptide subunit, it was concluded that the $\mathrm{N}$-terminal glutamic acid residue of the interpeptide bridge is of the L-configuration. Therefore, the peptidoglycan type of strain YIM $70085^{\mathrm{T}}$ was A4 $\alpha$, L-Lys-Gly-LGlu. Whole-cell sugars consisted of xylose, mannose and galactose. Phospholipids were diphosphatidylglycerol, phosphatidylglycerol, one unknown phospholipid (which was close to phosphatidylcholine, but its reaction with Dragendorff reagent was negative), one unknown glycolipid and traces of phosphatidylinositol. Menaquinones were MK-8 (83 \%), MK-7 (12\%) and MK-9 (15\%). Cellular fatty acid results are indicated in the species description and Table 1. 
Table 1. Differential chemotaxonomic characteristics of strain YIM $70085^{\top}$ and related taxa

Taxa: 1, Yania gen. nov. (YIM 70085 ${ }^{\mathrm{T}}$ ); 2, Brevibacteriaceae (Brevibacterium); 3, Micrococcus; 4, Arthrobacter; 5, Kocuria; 6, Nesterenkonia; 7, Renibacterium; 8, Rothia; 9, Citricoccus. Abbreviations: $\mathrm{A}_{2} \mathrm{pm}$, diaminopimelic acid; $\mathrm{MCA}_{\mathrm{var}}$, variable monocarboxylic amino acid in the interpeptide bridge; DMDG, dimannosyldiacylglycerol; DPG, diphosphatidylglycerol; GL, unidentified glycolipid; PE, phosphatidylethanolamine; PG, phosphatidylglycerol; PI, phosphatidylinositol; PIM, phosphatidylinositol mannosides; PL, unidentified phospholipid. Abbreviations for menaquinones are exemplified by MK- $8\left(\mathrm{H}_{2}\right)$, a partially saturated menaquinone with one of eight isoprene units hydrogenated. Abbreviations for fatty acids are exemplified by ai- $\mathrm{C}_{15: 0}, 12$-methyltetradecanoic acid; $\mathrm{i}-\mathrm{C}_{15: 0}, 13$-methyltetradecanoic acid; $\mathrm{C}_{16: 0}$, hexadecanoic acid.

\begin{tabular}{|c|c|c|c|c|c|c|c|c|c|}
\hline \multirow[t]{2}{*}{ Characteristic } & \multirow[t]{2}{*}{1} & \multirow[t]{2}{*}{$2^{\star}$} & \multicolumn{7}{|c|}{ Micrococcaceae } \\
\hline & & & $3 \dagger$ & $4 \dagger$ & $5 \dagger$ & $6 \dagger$ & $7 \dagger$ & $8 \dagger$ & $9 \ddagger$ \\
\hline Diamino acid & L-Lys & meso- $\mathrm{A}_{2} \mathrm{pm}$ & L-Lys & L-Lys & L-Lys & L-Lys & L-Lys & L-Lys & L-Lys \\
\hline Interpeptide bridge & Gly-L-Glu & None & $\begin{array}{l}\text { Peptide subunit } \\
\text { or D-Asp }\end{array}$ & $\mathrm{MCA}_{\mathrm{var}}$ & $\mathrm{L}-\mathrm{Ala}_{3-4}$ & Gly-L-Glu & L-Ala-Gly & $\mathrm{L}-\mathrm{Ala}_{3}$ & Gly-L-Glu \\
\hline Predominant menaquinone(s) & $7,8,9$ & $8\left(\mathrm{H}_{2}\right)$ & $8,8\left(\mathrm{H}_{2}\right)$ & $9\left(\mathrm{H}_{2}\right) / 8,9$ & $7\left(\mathrm{H}_{2}\right), 8\left(\mathrm{H}_{2}\right)$ & 8,9 & 9,10 & 7 & $7\left(\mathrm{H}_{2}\right) / 8\left(\mathrm{H}_{2}\right) / 9\left(\mathrm{H}_{2}\right)$ \\
\hline Polar lipids & $\begin{array}{l}\text { DPG, PG, } \\
\text { PI, PL, GL }\end{array}$ & $\begin{array}{l}\text { DPG, PG, } \\
\text { DMDG }\end{array}$ & $\begin{array}{l}\text { DPG, PI, PG, } \\
\text { PL, GL }\end{array}$ & $\begin{array}{l}\text { DPG, PG, } \\
\text { PI, DMDG }\end{array}$ & $\begin{array}{c}\mathrm{DPG}, \mathrm{PG} \\
(\mathrm{PI}, \mathrm{L}, \mathrm{GL})\end{array}$ & $\begin{array}{c}\text { DPG, PI, } \\
\text { PG, PL, GL }\end{array}$ & DPG, GL & DPG, PG & $\begin{array}{l}\text { DPG, PG, } \\
\text { PI, PL, GL }\end{array}$ \\
\hline Major fatty acids & $\begin{array}{l}\mathrm{i}-\mathrm{C}_{15: 0} \\
\text { ai- } \mathrm{C}_{15: 0} \\
\text { ai- } \mathrm{C}_{17: 0}\end{array}$ & $\begin{array}{l}\text { ai- } C_{15: 0} \\
\text { ai- } C_{17: 0}\end{array}$ & $\begin{array}{l}\mathrm{i}-\mathrm{C}_{15: 0} \\
\text { ai- } \mathrm{C}_{15: 0}\end{array}$ & $\begin{array}{l}\mathrm{i}-\mathrm{C}_{15: 0} \\
\text { ai- } \mathrm{C}_{15: 0} \\
\mathrm{i}-\mathrm{C}_{16: 0}\end{array}$ & $\begin{array}{l}\text { ai- } C_{15: 0} \\
\text { ai- } C_{17: 0} \\
\text { i- } C_{16: 0}\end{array}$ & $\begin{array}{l}\text { ai- } \mathrm{C}_{15: 0} \\
\text { ai- } \mathrm{C}_{17: 0} \\
\text { i- } \mathrm{C}_{16: 0}\end{array}$ & $\begin{array}{l}\text { ai- } \mathrm{C}_{15: 0} \\
\text { ai- } \mathrm{C}_{17: 0}\end{array}$ & $\begin{array}{c}\text { ai- } C_{15: 0} \\
\text { ai- } C_{17: 0} \\
C_{16: 0}\end{array}$ & $\begin{array}{l}\text { ai- } C_{15: 0}, \text { ai- } C_{17: 0} \\
\quad i-C_{16: 0}, i-C_{15: 0}\end{array}$ \\
\hline DNA G $+\mathrm{C}$ content $(\mathrm{mol} \%)$ & 53-54 & $60-67$ & $69-76$ & $56-69$ & $66-75$ & $70-72$ & $52-54$ & 49-53 & 68 \\
\hline
\end{tabular}

${ }^{\star}$ Data taken from Collins (2001).

†Data taken from Stackebrandt \& Schumann (2000).

$\ddagger$ Data taken from Altenburger et al. (2002). 


\section{Molecular analyses}

Extraction of genomic DNA and amplification of $16 \mathrm{~S}$ rDNA were done as described by Cui et al. (2001). Multiple alignments of sequences from a broad selection of actinobacteria and calculations of sequence similarity levels were carried out by using CLUSTAL X (Thompson et al., 1997). A phylogenetic tree was constructed by using the neighbourjoining method of Saitou \& Nei (1987) from $K_{\text {nuc }}$ values (Kimura, 1980, 1983). The topology of the phylogenetic tree was evaluated by the bootstrap resampling method of Felsenstein ( 1985) with 1000 replicates.

Chromosomal DNA for determination of base composition was prepared by following the method of Marmur (1961). The G + C content of the DNA was determined to be $53.5 \mathrm{~mol} \%$ by using the thermal denaturation method of Marmur \& Doty (1962).

Almost-complete 16S rDNA sequence data of strain YIM $70085^{\mathrm{T}}$ (1503 bp) were determined. BLAST search results of strain YIM $70085^{\mathrm{T}}$ came from GenBank/EMBL/DDBJ and the Protein Data Bank. Reference sequences that contained unidentified and unpublished sequences were not included (Fig. 2). After elimination of all sites for which nucleotides were not determined in any sequences, $1461 \mathrm{nt}$ was compared. A tree depicting the phylogenetic relationships of strain YIM $70085^{\mathrm{T}}$ and its closest relatives is shown in Fig. 2. Strain YIM $70085^{\mathrm{T}}$ formed a distinct phylogenetic lineage between the families Micrococcaceae and Brevibacteriaceae in the suborder Micrococcineae, but was related much more closely to the Micrococcaceae. Highest sequence similarity values were found with sequences of species of the family Micrococcaceae, such as Arthrobacter cumminsii (X93354) (94.31\%) Arthrobacter albus (AJ243421) (94.38\%), Kocuria rosea (Y11330) $(93 \cdot 72 \%)$ and Kocuria polaris (AJ278868) (93.65\%).

\section{Taxonomic conclusion}

The results of $16 \mathrm{~S}$ rDNA sequence comparisons clearly demonstrate that strain YIM $70085^{\mathrm{T}}$ is a member of the suborder Micrococcineae. Strain YIM $70085^{\mathrm{T}}$ has many unique $16 \mathrm{~S}$ rDNA signature nucleotides, compared to other families of the suborder Micrococcineae, such as 140-223 (A-G), 142-221 (C-A), 615-625 (G-U), 839-874 (A-A) and 1134-1140 (A-U) (see Table 2). Thus, strain YIM $70085^{\mathrm{T}}$ may represent a novel family of the suborder Micrococcineae, based on the viewpoint of Stackebrandt et al. (1997).

From the phylogenetic tree, strain YIM $70085^{\mathrm{T}}$ formed a separate phylogenetic lineage between the families Micrococcaceae and Brevibacteriaceae, but was related much more closely to the former. Sequence similarity levels between strain YIM $70085^{\mathrm{T}}$ and type strains of the suborder Micrococcineae were $<94.3 \%$. In addition, some signature nucleotides of the 16S rDNA sequence of strain YIM $70085^{\mathrm{T}}$ were not included in those of the families Micrococcaceae and Brevibacteriaceae (Stackebrandt et al., 1997; Stackebrandt \& Schumann, 2000). The 16 S rDNA signature nucleotide data of strain YIM $70085^{\mathrm{T}}$ are shown in Table 2. The results of comparison of $16 \mathrm{~S}$ rDNA signature nucleotides between the two families and strain YIM $70085^{\mathrm{T}}$ are indicated in bold type (see Table 2).

Additionally, there are significant differences in chemotaxonomic characteristics between strain YIM $70085^{\mathrm{T}}$ and the phylogenetically most closely related genera of the families Micrococcaceae and Brevibacteriaceae (see Table 1). The type genus of the family Brevibacteriaceae, Brevibacterium, obviously differs from strain YIM $70085^{\mathrm{T}}$ in displaying meso- $\mathrm{A}_{2} \mathrm{pm}$ as the diagnostic diamino acid of the peptidoglycan and partially saturated menaquinones. Members of the genera Micrococcus, Arthrobacter and

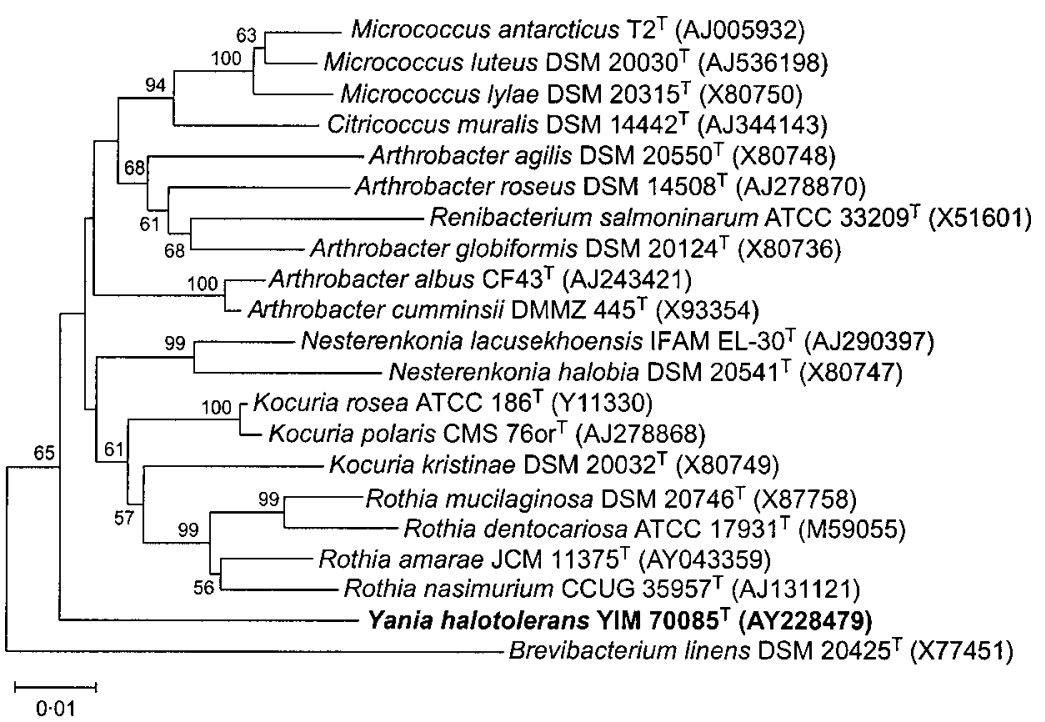

Fig. 2. Phylogenetic dendrogram obtained
by distance matrix analysis of $16 \mathrm{~S}$ rDNA
sequences, showing the position of strain
YIM $70085^{\top}$ among its phylogenetic neigh-
bours. Numbers on branch nodes are
bootstrap values (percentages of 1000
resamplings; only values $>50 \%$ are given).
The sequence of Streptomyces megasporus
DSM 41476 (GenBank accession no.
Z68100) was used as root. Bar, $1 \%$
sequence divergence. 
Table 2. Patterns of selected $16 \mathrm{~S}$ rDNA signature nucleotides that define families of the suborder Micrococcineae

Families: 1, Dermacoccaceae; 2, Rarobacteraceae; 3, Sanguibacteraceae; 4, Bogoriellaceae; 5, Dermatophilaceae; 6, Cellulomonadaceae; 7, Intrasporangiaceae; 8, Dermabacteraceae; 9, Jonesiaceae; 10, Microbacteriaceae, 11, Promicromonosporaceae; 12, Brevibacteriaceae; 13, Micrococcaceae; 14, Yania gen. nov. Data for Yania gen. nov. are from this study; data for all other taxa are from Stackebrandt \& Schumann (2000). R, Purine; $\mathrm{Y}$, pyrimidine; $\mathrm{V}$, variable. Residues in small capitals are present in some, but not all, strains. The results of comparison of signature nucleotides between the families Brevibacteriaceae and Micrococcaceae and strain YIM $70085^{\mathrm{T}}$ are indicated in bold type.

\begin{tabular}{|c|c|c|c|c|c|c|c|c|c|c|c|c|c|c|}
\hline Position & 1 & 2 & 3 & 4 & 5 & 6 & 7 & 8 & 9 & 10 & 11 & 12 & 13 & 14 \\
\hline $41-401$ & $\mathrm{G}-\mathrm{C}$ & $\mathrm{G}-\mathrm{C}$ & $\mathrm{G}-\mathrm{C}$ & $\mathrm{G}-\mathrm{C}$ & G-C & G-C & G-C & $\mathrm{G}-\mathrm{C}$ & $\mathrm{G}-\mathrm{C}$ & $\mathrm{G}-\mathrm{C}$ & G-C & $\mathrm{U}-\mathrm{A}$ & G-C & G-C \\
\hline $45-396$ & U-G & U-G & U-G & U-G & U-G & U-G & U-G & U-G & U-G & U-G & U-G & U-G & U-G & U-G \\
\hline 69-99 & $\mathrm{R}-\mathrm{U}$ & $\mathrm{A}-\mathrm{U}$ & $\mathrm{G}-\mathrm{U}$ & $\mathrm{A}-\mathrm{U}$ & $\mathrm{A}-\mathrm{U}$ & $\mathrm{G}-\mathrm{U}$ & $\mathrm{G}-\mathrm{U}$ & $\mathrm{A}-\mathrm{U}$ & $\mathrm{A}-\mathrm{U}$ & $\mathrm{R}-\mathrm{U}$ & $\mathrm{G}-\mathrm{U}$ & $\mathrm{C}-\mathrm{U}$ & $\mathrm{A}-\mathrm{U}$ & $\mathrm{C}-\mathrm{U}$ \\
\hline $144-178$ & $C-G$ & U-G & $C-G$ & U-G & U-A & C-G & $C-G$ & U-G & U-G & $C-G$ & U-G & U-G & C-G & C-G \\
\hline $140-223$ & $\mathrm{C}-\mathrm{G}, \mathrm{G}-\mathrm{C}$ & C-G & $\mathrm{G}-\mathrm{C}$ & G-U & C-G & C-G & G-Y & $C-G$ & $\mathrm{U}-\mathrm{U}$ & $\mathrm{G}-\mathrm{Y}$ & $C-G$ & G-C & $\mathrm{R}-\mathrm{U}$ & $A-G$ \\
\hline $142-221$ & C-G & $C-G$ & $\mathrm{C}-\mathrm{G}$ & $C-G$ & $C-G$ & $C-G$ & $C-G$ & $C-G$ & $C-G$ & U-A & $C-G$ & $\mathrm{U}-\mathrm{A}$ & C-G & C-A \\
\hline $248-276$ & $C-G$ & $\mathrm{C}-\mathrm{G}$ & $\mathrm{C}-\mathrm{G}$ & $C-G$ & $C-G$ & $C-G$ & $C-G$ & U-G & $C-G$ & $C-G$ & $C-G$ & $\mathrm{C}-\mathrm{C}$ & C-G & U-G \\
\hline $258-268$ & $\mathrm{G}-\mathrm{C}$ & $\mathrm{G}-\mathrm{C}$ & $\mathrm{A}-\mathrm{U}$ & $\mathrm{G}-\mathrm{C}$ & $\mathrm{A}-\mathrm{U}$ & $\mathrm{G}-\mathrm{C}$ & $\mathrm{A}-\mathrm{U}$ & $\mathrm{A}-\mathrm{U}$ & $\mathrm{G}-\mathrm{C}$ & $\mathrm{A}-\mathrm{U}$ & $\mathrm{G}-\mathrm{C}$ & G-C & $\mathrm{A}-\mathrm{U}$ & G-C \\
\hline 293-304 & G-Y & $\mathrm{G}-\mathrm{C}$ & $\mathrm{G}-\mathrm{C}$ & $\mathrm{G}-\mathrm{U}$ & G-U & $\mathrm{G}-\mathrm{C}$ & $\mathrm{G}-\mathrm{C}$ & $\mathrm{G}-\mathrm{C}$ & $\mathrm{G}-\mathrm{C}$ & $\mathrm{G}-\mathrm{U}$ & $\mathrm{G}-\mathrm{C}$ & G-C & G-U & G-U \\
\hline 379-384 & $\mathrm{C}-\mathrm{G}$ & $C-G$ & $\mathrm{C}-\mathrm{G}$ & $C-G$ & $C-G$ & $\mathrm{C}-\mathrm{G}$ & $C-G$ & $C-G$ & $\mathrm{G}-\mathrm{U}$ & $C-G$ & $C-G$ & $\mathrm{G}-\mathrm{C}$ & C-G & C-G \\
\hline 407-435 & $\mathrm{A}-\mathrm{U}, \mathrm{G}-\mathrm{C}$ & $\mathrm{A}-\mathrm{U}$ & $\mathrm{A}-\mathrm{U}$ & $\mathrm{A}-\mathrm{U}$ & $\mathrm{A}-\mathrm{U}$ & C-G & $\mathrm{A}-\mathrm{U}$ & $\mathrm{G}-\mathrm{Y}$ & $\mathrm{G}-\mathrm{C}$ & $\mathrm{A}-\mathrm{U}$ & $\mathrm{A}-\mathrm{U}$ & C-G & $\mathrm{A}-\mathrm{U}$ & $\mathrm{A}-\mathrm{U}$ \\
\hline $502-543$ & $\mathrm{~A}-\mathrm{U}$ & $\mathrm{G}-\mathrm{C}$ & $\mathrm{G}-\mathrm{C}$ & $\mathrm{G}-\mathrm{C}$ & $\mathrm{A}-\mathrm{U}$ & $\mathrm{G}-\mathrm{C}$ & $\mathrm{A}-\mathrm{U}$ & $\mathrm{R}-\mathrm{Y}$ & $\mathrm{G}-\mathrm{C}$ & $\mathrm{R}-\mathrm{Y}$ & $\mathrm{G}-\mathrm{C}$ & $\mathrm{A}-\mathrm{U}$ & R-Y & $\mathrm{A}-\mathrm{U}$ \\
\hline 586-755 & $\mathrm{C}-\mathrm{G}$ & U-G & $\mathrm{C}-\mathrm{G}$ & $C-G$ & $\mathrm{C}-\mathrm{G}$ & $C-G$ & $\mathrm{Y}-\mathrm{R}$ & U-A & $\mathrm{C}-\mathrm{G}$ & $C-G$ & $\mathrm{C}-\mathrm{G}$ & $\mathrm{U}-\mathrm{A}$ & C-G & C-G \\
\hline 589-650 & $\mathrm{U}-\mathrm{A}$ & U-A & $\mathrm{U}-\mathrm{A}$ & C-G & $\mathrm{U}-\mathrm{G}$ & U-A & U-A & $\mathrm{U}-\mathrm{G}$ & $\mathrm{U}-\mathrm{G}$ & U-A & U-A & U-A & C-G & U-G \\
\hline $591-648$ & U-A & U-A & U-A & U-A & U-A & U-A & U-A & U-A & U-A & U-A & U-A & G-U & U-A & U-A \\
\hline 610 & $\mathrm{R}$ & A & $\mathrm{U}$ & A & A & A & A & A & $\mathrm{U}$ & $\mathrm{V}$ & $\mathrm{U}$ & $\mathbf{A}$ & G & G \\
\hline 602-636 & $\mathrm{C}-\mathrm{G}$ & G-U & G-U & $C-G$ & $\mathrm{C}-\mathrm{G}$ & $\mathrm{C}-\mathrm{G}$ & $\mathrm{C}-\mathrm{G}$ & $C-G$ & U-G & $C-G$ & G-U & C-G & C-G & C-G \\
\hline $612-628$ & Y-G & U-A & U-A & $C-G$ & $C-G$ & $C-G$ & $C-G$ & Y-G & $C-G$ & $C-G$ & $C-G$ & G-C & C-G & U-A \\
\hline $615-625$ & $\mathrm{G}-\mathrm{C}$ & C-G & $\mathrm{A}-\mathrm{U}$ & $\mathrm{G}-\mathrm{C}$ & $\mathrm{G}-\mathrm{C}$ & $\mathrm{A}-\mathrm{U}$ & $\mathrm{R}-\mathrm{Y}$ & $\mathrm{A}-\mathrm{U}$ & $\mathrm{A}-\mathrm{U}$ & $\mathrm{A}-\mathrm{U}$ & $\mathrm{U}-\mathrm{A}$ & $\mathrm{A}-\mathrm{U}$ & G-C & G-U \\
\hline 616-624 & G-Y & $\mathrm{G}-\mathrm{C}$ & $\mathrm{G}-\mathrm{C}$ & $\mathrm{G}-\mathrm{C}$ & $\mathrm{G}-\mathrm{C}$ & $\mathrm{G}-\mathrm{C}$ & $\mathrm{G}-\mathrm{C}$ & $\mathrm{G}-\mathrm{C}$ & U-G & $\mathrm{G}-\mathrm{C}$ & $\mathrm{G}-\mathrm{U}$ & C-G & G-Y & G-C \\
\hline 660-745 & $\mathrm{G}-\mathrm{C}$ & $\mathrm{G}-\mathrm{C}$ & G-C & $\mathrm{G}-\mathrm{C}$ & G-C & G-C & $\mathrm{G}-\mathrm{C}$ & $\mathrm{G}-\mathrm{C}$ & $\mathrm{G}-\mathrm{C}$ & $\mathrm{G}-\mathrm{C}$ & U-A & $\mathrm{A}-\mathrm{U}$ & $\mathrm{v}$ & $\mathrm{A}-\mathrm{U}$ \\
\hline 668-738 & $\mathrm{A}-\mathrm{U}$ & $\mathrm{A}-\mathrm{U}$ & U-A & $\mathrm{A}-\mathrm{U}$ & $\mathrm{A}-\mathrm{U}$ & $\mathrm{U}-\mathrm{A}$ & $\mathrm{A}-\mathrm{U}$ & $\mathrm{A}-\mathrm{U}$ & U-A & $\mathrm{A}-\mathrm{U}$ & $\mathrm{A}-\mathrm{U}$ & $\mathrm{A}-\mathrm{U}$ & $\mathrm{A}-\mathrm{U}$ & $\mathrm{A}-\mathrm{U}$ \\
\hline 670-736 & $\mathrm{A}-\mathrm{U}$ & $\mathrm{A}-\mathrm{U}$ & $\mathrm{A}-\mathrm{U}$ & $\mathrm{A}-\mathrm{U}$ & $\mathrm{A}-\mathrm{U}$ & $\mathrm{A}-\mathrm{U}$ & $\mathrm{A}-\mathrm{U}$ & $\mathrm{A}-\mathrm{U}$ & $\mathrm{A}-\mathrm{U}$ & $\mathrm{A}-\mathrm{U}$ & $\mathrm{A}-\mathrm{U}$ & U-A & $\mathrm{A}-\mathrm{U}$ & $\mathrm{A}-\mathrm{U}$ \\
\hline 839-847 & U-A & U-A & U-A & $C-G$ & $C-G$ & $C-G$ & U-A & $\mathrm{R}-\mathrm{U}$ & U-A & $\mathrm{G}-\mathrm{U}$ & $C-G$ & $\mathrm{~A}-\mathrm{U}$ & $\mathrm{A}-\mathrm{U}$ & A-A \\
\hline 863 & $\mathrm{U}$ & A & $\mathrm{U}$ & $\mathrm{U}$ & $\mathrm{U}$ & A & $\mathrm{U}$ & $\mathrm{U}$ & $\mathrm{U}$ & $\mathrm{U}$ & $\mathrm{U}$ & $\mathbf{U}$ & $\mathrm{U}$ & $\mathrm{U}$ \\
\hline $1133-1141$ & $\mathrm{~A}-\mathrm{U}$ & $\mathrm{G}-\mathrm{C}$ & $\mathrm{A}-\mathrm{U}$ & $\mathrm{A}-\mathrm{U}$ & $\mathrm{A}-\mathrm{U}$ & $\mathrm{G}-\mathrm{C}$ & $\mathrm{A}-\mathrm{U}$ & $\mathrm{A}-\mathrm{U}$ & $\mathrm{A}-\mathrm{U}$ & $\mathrm{A}-\mathrm{U}$ & $\mathrm{G}-\mathrm{C}$ & $\mathrm{A}-\mathrm{U}$ & $\mathrm{A}-\mathrm{U}$ & $\mathrm{A}-\mathrm{U}$ \\
\hline $1134-1140$ & $C-G$ & $\mathrm{G}-\mathrm{C}$ & $\mathrm{C}-\mathrm{G}$ & $C-G$ & $\mathrm{C}-\mathrm{G}$ & $\mathrm{G}-\mathrm{C}$ & $\mathrm{C}-\mathrm{G}$ & $\mathrm{C}-\mathrm{G}$ & $\mathrm{C}-\mathrm{G}$ & $C-G$ & $\mathrm{G}-\mathrm{C}$ & C-G & C-G & $\mathrm{A}-\mathrm{U}$ \\
\hline $1244-1293$ & $C-G$ & $\mathrm{C}-\mathrm{G}$ & $\mathrm{C}-\mathrm{G}$ & $C-G$ & $C-G$ & $C-G$ & $\mathrm{C}-\mathrm{G}$ & $\mathrm{C}-\mathrm{G}$ & $\mathrm{C}-\mathrm{G}$ & $C-G$ & $\mathrm{C}-\mathrm{G}$ & U-A & C-G & C-G \\
\hline $1254-1283$ & $\mathrm{G}-\mathrm{C}$ & $\mathrm{G}-\mathrm{C}$ & $\mathrm{G}-\mathrm{C}$ & $\mathrm{G}-\mathrm{C}$ & $\mathrm{U}-\mathrm{A}$ & $\mathrm{G}-\mathrm{C}$ & $\mathrm{G}-\mathrm{C}$ & $\mathrm{G}-\mathrm{C}$ & $\mathrm{G}-\mathrm{C}$ & $\mathrm{G}-\mathrm{C}$ & $\mathrm{G}-\mathrm{C}$ & $\mathrm{A}-\mathrm{C}$ & G-C & G-C \\
\hline $1263-1272$ & $\mathrm{~A}-\mathrm{U}$ & $\mathrm{A}-\mathrm{U}$ & $\mathrm{A}-\mathrm{U}$ & $\mathrm{A}-\mathrm{U}$ & $\mathrm{A}-\mathrm{U}$ & $\mathrm{A}-\mathrm{U}$ & $\mathrm{A}-\mathrm{U}$ & $\mathrm{A}-\mathrm{U}$ & $\mathrm{A}-\mathrm{U}$ & $\mathrm{A}-\mathrm{U}$ & $\mathrm{A}-\mathrm{U}$ & C-G & $\mathrm{A}-\mathrm{U}$ & $\mathrm{A}-\mathrm{U}$ \\
\hline $1310-1327$ & $\mathrm{G}-\mathrm{C}$ & $\mathrm{G}-\mathrm{C}$ & $\mathrm{G}-\mathrm{C}$ & $\mathrm{G}-\mathrm{C}$ & $\mathrm{G}-\mathrm{C}$ & $\mathrm{G}-\mathrm{C}$ & $\mathrm{G}-\mathrm{C}$ & $\mathrm{G}-\mathrm{C}$ & $\mathrm{G}-\mathrm{C}$ & $\mathrm{A}-\mathrm{U}$ & $\mathrm{G}-\mathrm{C}$ & U-A & R-Y & G-C \\
\hline $1414-1486$ & $C-G$ & C-G & $C-G$ & C-G & $\mathrm{C}-\mathrm{G}$ & U-G & $C-G$ & C-G & C-G & U-A & $\mathrm{C}-\mathrm{G}$ & C-G & C-G & C-G \\
\hline
\end{tabular}

Kocuria in the family Micrococcaceae show highest $16 \mathrm{~S}$ rDNA sequence similarity to strain YIM $70085^{\mathrm{T}}$, but they can be differentiated easily, e.g. by partially saturated menaquinones, higher DNA G $+\mathrm{C}$ contents and different interpeptide bridges of the peptidoglycan. Peptidoglycan type L-Lys-Gly-L-Glu was found previously in the type strain of Nesterenkonia halobia. However, the combination of this peptidoglycan type with the menaquinone profile and polar lipid and fatty acid patterns of strain YIM $70085^{\mathrm{T}}$, as well as its $\mathrm{G}+\mathrm{C}$ content, are unique among members of the suborder Micrococcineae.

Thus, based on these results, which show that strain YIM $70085^{\mathrm{T}}$ differs from all established genera of the suborder Micrococcineae in rDNA signature nucleotides, $16 \mathrm{~S}$ rDNA sequence and phenotypic characteristics, a novel genus and species, Yania halotolerans gen. nov., sp. nov., are proposed to accommodate isolate YIM $70085^{\mathrm{T}}$. However, as the novel genus and species are represented only by a single strain, affiliation to a novel higher taxon should await phylogenetic analyses of further isolates of this genus.

\section{Description of Yania gen. nov.}

Yania [Ya' ni.a. N.L. fem. n. Yania named after Sun-Chu Yan (1912-1994), a Chinese microbiologist who devoted his life to the study of actinomycete taxonomy and antibiotics].

Cells are non-motile, aerobic, Gram-positive, non-sporeforming, coccoid or oval and about $0 \cdot 4-0 \cdot 7 \mu \mathrm{m}$ in diameter and occur singly or in clusters. Oxidase-negative and 
catalase-positive. Cell-wall peptidoglycan type is $\mathrm{A} 4 \alpha$, L-Lys-Gly-L-Glu. Whole-cell sugars are xylose, mannose and galactose. Phospholipids are diphosphatidylglycerol, phosphatidylglycerol, one unknown phospholipid, one unknown glycolipid and traces of phosphatidylinositol. Predominant menaquinones are MK-8, MK-7 and MK-9. Major cellular fatty acids are $\mathrm{i}-\mathrm{C}_{15: 0}$, ai- $\mathrm{C}_{15: 0}$ and ai- $\mathrm{C}_{17: 0}$. DNA G $+\mathrm{C}$ content is $53-54 \mathrm{~mol} \%$. The type species is Yania halotolerans.

\section{Description of Yania halotolerans sp. nov.}

Yania halotolerans (ha.lo.to'le.rans. Gr. n. hals salt; L. pres. part. tolerans tolerating; N.L. part. adj. halotolerans referring to the organism's ability to tolerate high salt concentrations).

Morphological, chemotaxonomic and general characteristics are as described for the genus. Colonies reach maximum size $\left(2 \mathrm{~mm}\right.$ in diameter) after 1 week incubation at $28^{\circ} \mathrm{C}$. They are yellow, circular, lubricious and opaque. Almost all carbon sources tested are utilized, including glucose, galactose, arabinose, starch, cellobiose, lactose, mannose, mannitol, fructose, sucrose, maltose and xylose, but acid is produced only from glucose, maltose, sucrose and fructose. Positive for milk peptonization and urease, but negative for milk coagulation, nitrate reduction, gelatin liquefaction, growth on cellulose and production of $\mathrm{H}_{2} \mathrm{~S}$ and melanin. Starch and Tweens 20, 40 and 80 are not hydrolysed. Cells do not form indole; methyl red and VogesProskauer tests are negative. Temperature range for growth is $10-40{ }^{\circ} \mathrm{C}$, with an optimum temperature of $28-30{ }^{\circ} \mathrm{C}$. Growth $\mathrm{pH}$ is optimal at $7 \cdot 0-8 \cdot 0$. Concentration ranges of $\mathrm{NaCl}, \mathrm{KCl}$ and $\mathrm{MgCl}_{2} \cdot 6 \mathrm{H}_{2} \mathrm{O}$ for growth are $0-25,0-20$ and $0-15 \%$, respectively. Cell-wall peptidoglycan type is $\mathrm{A} 4 \alpha$, L-Lys-Gly-L-Glu. Whole-cell sugars consist of glucose, arabinose, xylose and ribose. Phospholipids are diphosphatidylglycerol, phosphatidylglycerol, one unknown phospholipid, one unknown glycolipid and traces of phosphatidylinositol. Menaquinones are MK-8 (83\%), MK-7 (12\%) and MK-9 (15\%). Cellular fatty acids are i- $C_{15: 0}(44 \cdot 29 \%)$, ai- $C_{15: 0}(35 \cdot 60 \%)$, ai- $\mathrm{C}_{17: 0}(9 \cdot 74 \%)$, $\mathrm{C}_{15: 0}(0 \cdot 28 \%), \mathrm{C}_{16: 0}(0 \cdot 87 \%), \mathrm{i}-\mathrm{C}_{14: 0}(1 \cdot 55 \%), \mathrm{i}-\mathrm{C}_{16: 0}$ $(3 \cdot 35 \%)$, i-C $17: 0(2 \cdot 76 \%)$, ai- $\mathrm{C}_{19: 0}(0 \cdot 42 \%), \mathrm{C}_{16: 1} \omega 7 c$ $(0 \cdot 48 \%)$ and $\mathrm{i}-\mathrm{C}_{15: 1}(0 \cdot 65 \%)$. DNA $\mathrm{G}+\mathrm{C}$ content is $53 \cdot 5 \mathrm{~mol} \%$.

The type strain is YIM $70085^{\mathrm{T}}\left(=\right.$ CCTCC AA001023 $3^{\mathrm{T}}=$ DSM $\left.15476^{\mathrm{T}}\right)$. Isolated from saline soil that was collected in Xinjiang Province, west China.

\section{Acknowledgements}

We thank H. G. Trüper and J. Euzéby for their valuable advice on nomenclature and etymology. This research was supported by the Ministry of Science and Technology, P.R. China (project no. 2001CCC00600), the National Natural Science Foundation of China (project no. 30270004), Yunnan Provincial Natural Science Foundation (project no. 20001C001Q) and Yunnan Education Commission Foundation.

\section{References}

Altenburger, P., Kämpfer, P., Schumann, P., Steiner, R., Lubitz, W. \& Busse, H.-J. (2002). Citricoccus muralis gen. nov., sp. nov., a novel actinobacterium isolated from a medieval wall painting. Int J Syst Evol Microbiol 52, 2095-2100.

Breed, R. S. (1953). The families developed from Bacteriaceae Cohn with a description on the family Brevibacteriaceae. VI Congresso Internazionale Microbiologia Roma 1, 10-15.

Collins, M. D. (2001). The genus Brevibacterium. In The Prokaryotes: an Evolving Electronic Resource for the Microbiological Community, 3rd edn, release 3.6, 22 June 2001. Edited by M. Dworkin, N. Falkow, H. Rosenberg, K-H. Schleifer \& E. Stackebrandt. New York: Springer (available online at http://link.springer-ny.com/link/service/books/ 10125/).

Collins, M. D. \& Jones, D. (1980). Lipids in the classification and identification of coryneform bacteria containing peptidoglycans based on 2,4-diaminobutyric acid. J Appl Bacteriol 48, 459-470.

Collins, M. D., Pirouz, T., Goodfellow, M. \& Minnikin, D. E. (1977). Distribution of menaquinones in actinomycetes and corynebacteria. J Gen Microbiol 100, 221-230.

Cui, X.-L., Mao, P.-H., Zeng, M., Li, W.-J., Zhang, L.-P., Xu, L.-H. \& Jiang, C.-L. (2001). Streptimonospora salina gen. nov., sp. nov., a new member of the family Nocardiopsaceae. Int J Syst Evol Microbiol 51, 357-363.

Felsenstein, J. (1985). Confidence limits on phylogenies: an approach using the bootstrap. Evolution 39, 783-791.

Frank, H., Rettenmeier, A., Weicker, H., Nicholson, G. J. \& Bayer, E. (1980). A new gas chromatographic method for determination of amino acid levels in human serum. Clin Chim Acta 105, 201-211.

Groth, I., Schumann, P., Rainey, F. A., Martin, K., Schuetze, B. \& Augsten, K. (1997). Demetria terragena gen. nov., sp. nov., a new genus of actinomycetes isolated from compost soil. Int $J$ Syst Bacteriol 47, 1129-1133.

Kämpfer, P., Steiof, M. \& Dott, W. (1991). Microbiological characterization of a fuel oil contaminated site including numerical identification of heterotrophic water and soil bacteria. Microb Ecol 21, 227-251.

Kelly, K. L. (1964). Inter-Society Color Council-National Bureau of Standards Color-Name Charts Illustrated with Centroid Colors. Washington, DC: US Government Printing Office.

Kimura, M. (1980). A simple method for estimating evolutionary rates of base substitutions through comparative studies of nucleotide sequences. J Mol Evol 16, 111-120.

Kimura, M. (1983). The Neutral Theory of Molecular Evolution. Cambridge: Cambridge University Press.

Kroppenstedt, R. M. (1985). Fatty acid and menaquinone analysis of actinomycetes and related organisms. In Chemical Methods in Bacterial Systematics, pp. 173-199. Edited by M. Goodfellow \& D. E. Minnikin. London: Academic Press.

MacKenzie, S. L. (1987). Gas chromatographic analysis of amino acids as the $N$-heptafluorobutyryl isobutyl esters. J Assoc Off Anal Chem 70, 151-160.

Marmur, J. (1961). A procedure for the isolation of deoxyribonucleic acid from microorganisms. J Mol Biol 3, 208-218.

Marmur, J. \& Doty, P. (1962). Determination of the base composition of deoxyribonucleic acid from its thermal denaturation temperature. J Mol Biol 5, 109-118.

Meier, A., Kirschner, P., Schröder, K.-H., Wolters, J., Kroppenstedt, R. M. \& Böttger, E. C. (1993). Mycobacterium intermedium sp. nov. Int J Syst Bacteriol 43, 204-209. 
Minnikin, D. E., Collins, M. D. \& Goodfellow, M. (1979). Fatty acid and polar lipid composition in the classification of Cellulomonas, Oerskovia and related taxa. J Appl Bacteriol 47, 87-95.

Pribram, E. (1929). A contribution to the classification of microorganisms. J Bacteriol 18, 361-394.

Saitou, N. \& Nei, M. (1987). The neighbor-joining method: a new method for reconstructing phylogenetic trees. Mol Biol Evol 4, 406-425.

Schleifer, K. H. (1985). Analysis of the chemical composition and primary structure of murein. Methods Microbiol 18, 123-156.

Schleifer, K. H. \& Kandler, O. (1972). Peptidoglycan types of bacterial cell walls and their taxonomic implications. Bacteriol Rev 36, 407-477.

Shirling, E. B. \& Gottlieb, D. (1966). Methods for characterization of Streptomyces species. Int J Syst Bacteriol 16, 313-340.
Stackebrandt, E. \& Schumann, P. (2000). Description of Bogoriellaceae fam. nov., Dermacoccaceae fam. nov., Rarobacteraceae fam. nov. and Sanguibacteraceae fam. nov. and emendation of some families of the suborder Micrococcineae. Int J Syst Evol Microbiol 50, 1279-1285.

Stackebrandt, E., Rainey, F. A. \& Ward-Rainey, N. L. (1997). Proposal for a new hierarchic classification system, Actinobacteria classis nov. Int J Syst Bacteriol 47, 479-491.

Stanek, J. L. \& Roberts, G. D. (1974). Simplified approach to identification of aerobic actinomycetes by thin-layer chromatography. Appl Microbiol 28, 226-231.

Thompson, J. D., Gibson, T. J., Plewniak, F., Jeanmougin, F. \& Higgins, D. G. (1997). The CLUSTAL_X windows interface: flexible strategies for multiple sequence alignment aided by quality analysis tools. Nucleic Acids Res 25, 4876-4882. 